\title{
Sirtuin1 single nucleotide polymorphism (A2191G) is a diagnostic marker for vibration-induced white finger disease
}

\author{
Susanne Voelter-Mahlknecht ${ }^{1,2^{*}}$, Bernd Rossbach ${ }^{1}$, Christina Schleithoff ${ }^{3}$, Christian L Dransfeld ${ }^{3}$,
} Stephan Letzel ${ }^{1}$ and Ulrich Mahlknecht ${ }^{3}$

\begin{abstract}
Background: Vibration-induced white finger disease (VWF), also known as hand-arm vibration syndrome, is a secondary form of Raynaud's disease, affecting the blood vessels and nerves. So far, little is known about the pathogenesisof the disease. WWF is associated with an episodic reduction in peripheral blood flow. Sirtuin 1, a class III histone deacetylase, has been described to regulate the endothelium dependent vasodilation by targeting endothelial nitric oxide synthase. We assessed Sirt1 single nucleotide polymorphisms in patients with VWF to further elucidate the role of sirtuin 1 in the pathogenesis of WWF.

Methods: Peripheral blood samples were obtained from 74 patients with WWF (male 93.2\%, female 6.8\%, median age 53 years) and from 317 healthy volunteers (gender equally distributed, below 30 years of age). Genomic DNA was extracted from peripheral blood mononuclear cells and screened for potential Sirt1single nucleotide polymorphisms. Four putative genetic polymorphisms out of 113 within the Sirt1 genomic region (NCBI Gene Reference: NM_012238.3) were assessed. Allelic discrimination was performed by TaqMan-polymerasechainreaction-based allele-specific genotyping single nucleotide polymorphism assays.
\end{abstract}

Results: Sirt1single nucleotide polymorphism A2191G (Assay C_25611590_10, rs35224060) was identified within Sirt1 exon 9 (amino acid position 731, lle $\rightarrow \mathrm{Val}$ ), with differing allelic frequencies in the WWF population (A/A: 70.5\%, A/G: 29.5\%, G/G: 0\%) and the control population (A/A: 99.7\%, A/G: 0.3\%, G/G: 0.5\%), with significance levels of $P<0.001$ (Mann-Whitney $U$ test (two-tailed) $P<0.001$; F-exact $t$-test and Chi-square test with Yates correction (all two-tailed): $P<0.0001)$. The heterogeneous $A / G$ genotype in base pair position 2191 is significantly overrepresented in the WW patient population when compared with healthy controls.

Conclusion: We identified theSirt1 ${ }_{\text {A2191G }}$ Single nucleotide polymorphism as a diagnostic marker for WWF.

Keywords: Epigenetics, Hand-arm vibration syndrome (HAVS), Sirtuins, Vibration-induced white finger disease (MWF)

\section{Background}

Vibration-induced white finger disease (VWF) is an industrial injury that is triggered by the continued use of vibrating hand-held machinery. The disease is a widespread and officially recognized occupational disease affecting tens of thousands of employees. According to

\footnotetext{
* Correspondence: susanne.voelter-mahlknecht@med.uni-tuebingen.de ${ }^{1}$ Institute of Occupational, Social and Environmental Health, University of Mainz, Obere Zahlbacher Strasse 67, D-55131 Mainz, Germany

${ }^{2}$ Institute of Occupational Medicine, Social Medicine and Health Services Research, University of Tuebingen, Wilhelmstrasse 27, D-72074 Tuebingen, Germany

Full list of author information is available at the end of the article
}

data that have been published by the Medical Research Council, around 2 million people in Britain are continuously subjected to potentially harmful levels of handarm vibration and around 300,000 people are anticipated to suffer from moderate to severe finger blanching (VWF) linked to such exposure, which may lead to considerable time off work, early retirement and considerable payouts from civil compensation schemes. In fact, a UK government fund that had been set up to cover claims by ex-coalminers who were exposed to the use of vibrating hand-held machinery had exceeded $£ 100$ million in payments by 2004 [1].

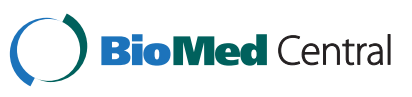




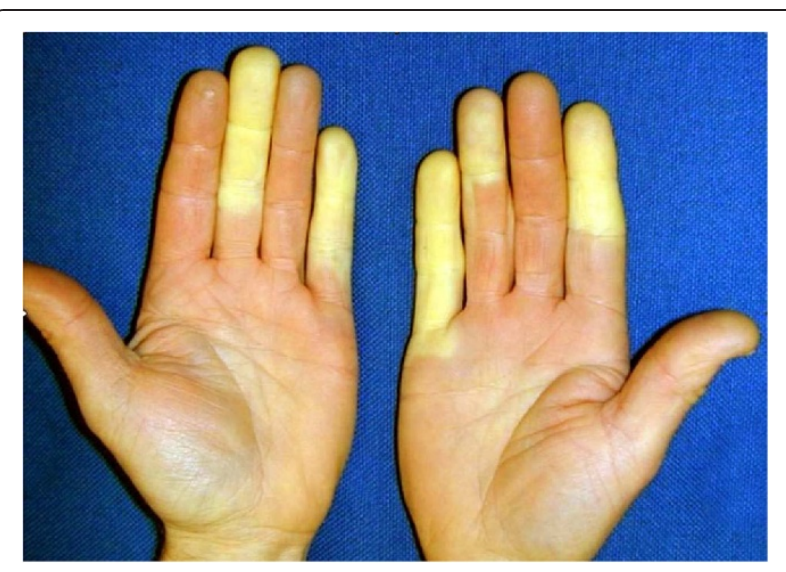

Figure 1 Hands of a person suffering from vibration-induced white finger disease.

VWF is characterized by vasospastic attacks and a cold sensation in the fingers followed by cyanotic discoloration or skin pallor (Figure 1) [2-5]. In addition, the sensitivity in the affected fingers is usually reduced. As a consequence, some of the affected individuals have difficulty carrying out manual activities because of their limited fine motor skills. Attacks may be associated with a tingling sensation, feeling loss, stiffness and at times even severe pain. In an early stage of the disease, vasospastic attacks occur particularly under the influence of low temperature. In later stages of the disease, a cold ambient temperature is no longer required as a trigger to cause vasospasm. VWFmay be triggered by hand-operated technical tools and machines that cause high frequency vibration with an oscillation rate $>50 \mathrm{~Hz}$. The occurrence of the disease depends on the length and intensity of daily exposure to vibration. To date, little is known about individual susceptibility factors with respect to VWF.

The pathogenesis of this disease is currently unclear. Epigenetics is gaining increasing importance in the understanding of numerous diseases. Epigenetic pathways have recently been suggested to be important in the regulation of vascular gene expression in the pathophysiology of atherosclerosis [6,7], the microvascular environment of tumors [8], cytokine-inducible gene expression in vascular endothelium [9], and in the developmental regulation of vascular remodeling $[10,11]$. Chromatin-based regulatory mechanisms may therefore play a key role in the constitutive expression of endothelium-restricted genes [10].

A single nucleotide polymorphism (SNP) is defined as the difference between chromosomes in the base present at a particular site in the DNA sequence that naturally occurs within a population, and presents the most common type (90\%) of genetic variation in humans [12]. Hopefully, increasing knowledge of an individual's SNP genotype may contribute to the assessment of disease susceptibility and individualized treatment modalities [13].
Based on structural and functional similarities, mammalian histone deacetylases (HDACs) are grouped into four categories. There are three classes of non-sirtuin HDACs, comprising the yeast HDACright parietal dorsal 3 homologs (class I HDACs); class II HDACs, which share a significant degree of homology with the yeast HDA1; and the most recently described class IV HDACs, which comprise HDAC11-related enzymes. There is one class of sirtuin HDACs (class III HDACs), which are homologs to the yeast Sir2 protein.

The yeast Sir2 protein has seven human homologs (SIRT1-7), which play a central role in epigenetic gene silencing, DNA repair and recombination, cell-cycle, microtubule organization, and in the regulation of aging [14].

SIRT1, which is a member of the Sir2 family of $\mathrm{NAD}^{+}$-dependent HDACs, deacetylates histone $\mathrm{H} 3$ lysines 9 and 14 and specifically histone H4 lysine-16, while it hydrolyzes one molecule of $\mathrm{NAD}^{+}$for every lysine residue that is deacetylated [14,15]. Derivatives of the yeast Sir2 HDAC share a common catalytic domain, which is highly conserved in organisms ranging from bacteria to humans and which is composed of two distinct motifs that bind $\mathrm{NAD}^{+}$and the acetyllysine substrate, respectively [14-17]. SIRT1 is known to directly modify chromatin and to silence transcription, to modulate the meiotic checkpoint, and as a probable antiaging effect, to increase genomic stability and to suppress recombinant DNA recombination $[18,19]$. While, for yeast Sir2, no targets are known apart from histones, SIRT1 has a large and still growing list of targets,including p53 and forkhead transcription factors, which are mammalian homologs of Daf-16 and which are known to function as sensors of the insulin signaling pathway $[14,18]$.

\section{Results and discussion}

Within the Sirt1 genomic sequence, 113 potential SNPs were identified, of which six were coding nonsynonymous SNPs. Three out of six were found to be false-positive SNPs, but four out of six were further analyzed in patients with VWF in relation to healthy controls. While rs1063114 and rs1063112 were in fact identified to be false positive SNPs, we confirmed rs1063111, rs35224060, rs3740051 and rs2236319 to be true SNPs. For rs1063111, we had identified a fre quency of $99.5 \%$ for the $\mathrm{T} / \mathrm{T}$ allele and $0.5 \%$ for the $\mathrm{A} / \mathrm{T}$ allele in the control population, which remained unchanged in the VWF patient population. Because the $\mathrm{T} / \mathrm{T}$ allele had a frequency of $98.3 \%$ versus $1.7 \%$ for the A/T allele in the HapMap data for Central Europeans, this SNP may be considered a true SNP, which remains unchanged within the VWF population. Important details regarding rs3740051, the Sirt1 
promoter SNP located 521 bp upstream of the translational start codon, also need to be mentioned.In the Central European Caucasian population, we identified a $90 \%$ frequency for the A/A genotype, $9.5 \%$ for the $\mathrm{A} / \mathrm{G}$ genotype and an allelic frequency of $0.5 \%$ for the $\mathrm{G} / \mathrm{G}$ genotype. This is in accordance with the HapMap data and showed no difference in the population of patients with VWF. However, the HapMap allelic reported for the Indian (A/A: 98.0\%, A/G: 1.0\%, G/G: 1.0\%), Chinese (A/A: $51.1 \%, A / G: 35.6 \%, G / G: 13.3 \%$ ) and Japanese (A/ A: $38.6 \%, \mathrm{~A} / \mathrm{G}: 56.8 \%$, G/G: $4.5 \%)$ populations is remarkable divergent, a fact that is currently unexplained. Similar observations have been made for the Sirt1 intron 4 SNP rs2236319: Central European Caucasian population -A/A: $90.0 \%$, A/G: $8.3 \%$, G/G: $1.7 \%$, with no difference between the control population and the VWF population; Chinese population- A/A: 51.1\%, A/G: 35.6\%, G/G: $13.3 \%$, which is identical to the rs 3740051 distribution; and Japanese population - A/A: 31.8\%, A/G: 63.6\%, G/G: $4.5 \%$, which is also noteworthy and currently without explanation. The most striking results have however been obtained for rs35224060, the Sirt1 SNP A2191G (Assay C_25611590_10) that was identified within Sirt1 exon 9 (amino acid position 731, Ile $\rightarrow$ Val), with differing allelic frequencies in the VWF population (A/A: 70.5\%, A/G: 29.5\%, G/G: 0\%) and the control population (A/A: 99.7\%, A/G: 0.3\%, G/G: 0.5\%) with significance levels of $P<0.001$ (Mann-Whitney $U$ test (two-tailed) $P<0.001 ; \quad F$-exact $t$-test and Chi-square test with Yates correction (all two-tailed): $P<0.0001$ respectively). The heterogeneous $\mathrm{A} / \mathrm{G}$ genotype in $\mathrm{bp}$ position 2191 is significantly overrepresented in the VWF patient population when compared with healthy controls (Table 1).

To date, little is known about the pathogenesis of VWF. It is however widely accepted that both the nervous and the vascular systems are affected and that vasoconstrictive effects dominate over vasodilatative effects during a vasospastic attack. In this process, endothelium-dependent and endothelium-independent mechanisms regulating the vascular tone may be distinguished. The endotheliumdependent vascular regulation is based on the interplay of competing vasoconstrictive and vasodilatative substrates, of which nitric oxide (NO), a factor that significantly depends on the activity of the endothelial isoform of NO synthase (eNOS, synonym: NOS3), is a key player.

There is increasing evidence that epigenetic mechanisms play a key role in a number of vascular disorders $[10,20]$. SIRT1 and eNOS co-localize and co-precipitate in endothelial cells, and SIRT1 deacetylates eNOS, thus stimulating eNOS activity, which subsequently increases endothelial NO [21]. Fully functional Sirt1 is essential in the regulation of endothelium-dependent vasodilation and may have profound effects not only on the control of the local vascular tone and systemic blood pressure, but also in the pathogenesis of VWF.

\section{Conclusions}

Our study shows an important, highly significant accumulation of the heterogeneous genotype of the Sirt1 SNP A2191G (Ile $\rightarrow$ Val) when compared with healthy controls $(P<0.001)$.

Table 1 Comparison of the allelic distribution of patients with vibration-induced white finger disease and healthy controls

\begin{tabular}{|c|c|c|c|c|c|c|}
\hline \multirow{2}{*}{$\frac{\text { dbSNP [Assay ID] }}{\text { rs1063114 }}$} & \multicolumn{3}{|c|}{ Patients with VWF } & \multicolumn{3}{|c|}{ Healthy controls } \\
\hline & Allele A/A: 0/74 & Allele A/T: 0/74 & Allele T/T: 74/74 & Allele A/A: 0/203 & Allele A/T: 0/203 & Allele T/T: 203/203 \\
\hline [C_9638456_10] & $(0 \%)$ & $(0 \%)$ & $(100 \%)$ & $(0 \%)$ & $(0 \%)$ & $(100 \%)$ \\
\hline rs1063111 & Allele A/A: 0/74 & Allele A/T: 0/74 & Allele T/T: 74/74 & Allele A/A: 0/200 & Allele A/T: 1/200 & Allele T/T: 199/200 \\
\hline [C_9638445_10] & $(0 \%)$ & $(0 \%)$ & $(100 \%)$ & $(0 \%)$ & $(0.5 \%)$ & (99.5\%) \\
\hline rs1063112 & Allele C/C: 0/49 & Allele C/T: 0/49 & Allele T/T: 49/49 & Allele C/C: 0/299 & Allele C/T: 0/299 & Allele T/T: 299/299 \\
\hline [SIRT1-A485] & $(0 \%)$ & $(0 \%)$ & $(100 \%)$ & $(100 \%)$ & $(0 \%)$ & $(100 \%)$ \\
\hline rs35224060 & Allele A/A: 52/74 & Allele A/G: 22/74 & Allele G/G: 0/74 & Allele A/A: 316/317 & Allele A/G: 1/317 & Allele G/G: 0/317 \\
\hline [C_25611590_10] & $(70.5 \%)$ & $(29.5 \%)$ & $(0 \%)$ & (99.7\%) & $(0.3 \%)$ & $(0 \%)$ \\
\hline \multicolumn{7}{|l|}{ rs3740051 } \\
\hline [C_27471644_10] & Allele A/A: 62/68 & Allele A/G: 6/68 & Allele G/G: 0/68 & Allele A/A: 170/189 & Allele A/G: 18/189 & Allele G/G: 1/189 \\
\hline Sirt1 promoter & (91\%) & $(9 \%)$ & $(0 \%)$ & $(90 \%)$ & $(9,5 \%)$ & $(0.5 \%)$ \\
\hline \multicolumn{7}{|l|}{ rs2236319 } \\
\hline [C_15954063_10] & Allele A/A: 58/66 & Allele A/G: 8/66 & Allele G/G: 0/66 & Allele A/A: 171/193 & Allele A/G: 22/193 & Allele G/G: 0/193 \\
\hline Sirt1 Intron 4 & $(88 \%)$ & $(12 \%)$ & $(0 \%)$ & $(88 \%)$ & $(12 \%)$ & $(0 \%)$ \\
\hline
\end{tabular}

dbSNP: Database of Single Nucleotide Polymorphisms; VWF: vibration white finger disease. 
Table 2 Overview of the potential nonsynonymous Sirt1 SNPsin silico and analyzed

\begin{tabular}{|c|c|c|c|c|c|c|c|}
\hline $\begin{array}{l}\mathrm{dbSNP} \\
\text { [Assay ID] }\end{array}$ & Region & $5^{\prime}$ Near Seq 30 bp & Allele & $3^{\prime}$ Near Seq 30 bp & $\begin{array}{l}\text { Codon } \\
\text { position }\end{array}$ & $\begin{array}{l}\text { Protein } \\
\text { residue }\end{array}$ & $\begin{array}{c}\text { Amino } \\
\text { acid } \\
\text { position }\end{array}$ \\
\hline rs1063114 & \multirow[t]{2}{*}{ Exon 8} & \multirow[t]{2}{*}{ agactgtgatgtcataattaatgaattgtg } & $A / T$ & \multirow[t]{2}{*}{ cataggttaggtggtgaatatgccaaactt } & \multirow[t]{2}{*}{3} & \multirow{2}{*}{$\begin{array}{c}\text { Ter/Cys } \\
{[* / C]}\end{array}$} & \multirow[t]{2}{*}{490} \\
\hline [C_9638456_10] & & & $\begin{array}{c}\text { ancestral allele: } \\
\mathbf{T}\end{array}$ & & & & \\
\hline rs1063111 & \multirow[t]{2}{*}{ Exon 8} & \multirow[t]{2}{*}{ ttgatgtagagcttcttggagactgtgatg } & $A / T$ & \multirow[t]{2}{*}{ cataattaatgaattgtgtcataggttagg } & \multirow[t]{2}{*}{2} & \multirow{2}{*}{$\begin{array}{c}\text { Asp/Val } \\
{[\mathrm{D} / \mathrm{V}]}\end{array}$} & \multirow[t]{2}{*}{484} \\
\hline [C_9638445_10] & & & $\begin{array}{c}\text { ancestral allele: } \\
\mathbf{T}\end{array}$ & & & & \\
\hline rs1063112 & \multirow[t]{2}{*}{ Exon 8} & \multirow[t]{2}{*}{ atgtagagcttcttggagactgtgatgtca } & $\mathrm{C} / \mathrm{T}$ & \multirow[t]{2}{*}{ aattaatgaattgtgtcataggttaggtgg } & \multirow[t]{2}{*}{2} & Thr/lle & \multirow[t]{2}{*}{485} \\
\hline [SIRT1-A485] & & & $\begin{array}{c}\text { ancestral allele: } \\
\mathbf{T}\end{array}$ & & & {$[\mathrm{T} / \mathrm{I}]$} & \\
\hline rs35224060 & \multirow[t]{2}{*}{ Exon 9} & \multirow[t]{2}{*}{ ggagatgatcaagaggcaattaatgaagct } & $A / G$ & \multirow[t]{2}{*}{ tatctgtgaaacaggaagtaacagacatga } & \multirow[t]{2}{*}{1} & Ile/Val & \multirow[t]{2}{*}{731} \\
\hline [C_25611590_10] & & & $\begin{array}{c}\text { ancestral allele: } \\
\text { n.a. }\end{array}$ & & & {$[\mathrm{I} / \mathrm{V}]$} & \\
\hline rs3740051 & \multirow[t]{2}{*}{ promoter } & \multirow[t]{2}{*}{ agccgectccttttgectctcttcctactt } & $A / G$ & \multirow[t]{2}{*}{ ttaacaaaacagaacgactatccaacgtat } & \multirow[t]{2}{*}{-} & \multirow[t]{2}{*}{-} & \multirow[t]{2}{*}{-} \\
\hline [C_27471644_10] & & & $\begin{array}{c}\text { ancestral allele: } \\
\text { A }\end{array}$ & & & & \\
\hline rs2236319 & Intron 4 & agggatgtcagtctgatggagaaattgggt & $A / G$ & tttgttagatctttatgagaaactggaaac & - & - & - \\
\hline [C_15954063_10] & & & $\begin{array}{c}\text { ancestral allele: } \\
\text { A }\end{array}$ & & & & \\
\hline
\end{tabular}

bp: base pair; dbSNP: Database of Single Nucleotide Polymorphisms.

We therefore claim the $\operatorname{Sirt}_{2191}$ A/G genotype to be a risk factor for VWF, and that it may be used as a biological marker to facilitate the identification of risk populations who are being considered for exposure to potentially hazardous hand-held vibrating machinery. This may prevent thousands of employees from experiencing physical pain and disability, in addition to longlasting administrative fights for VWF compensation, which frequently end unsettled and in great frustration.

\section{Methods}

\section{Patients and control group}

Peripheral blood samples were obtained from 74 patients with VWF and from 317 healthy volunteers. Informed consent was given and the study was approved by the local ethics committee. The patient group was composed of 69 male (93.2\%) and five female (6.8\%) patients with a median age of 53 years, ranging from 29 to 74 years; the gender contribution is caused by a higher frequency on VWF in men and reflects the real world gender distribution of the disease. In the control group, genders were equally distributed (all below the age of 30 years). Genomic DNA was extracted from $5 \mathrm{~mL}$ peripheral EDTA (ethylenediaminetetraacetic acid)-blood with the FlexiGene DNA Kit (Qiagen, Hilden, Germany) according to the manufacturer's instructions. DNA quantification was carried out on a Nanodrop ND-1000 Spectrophotometer (Peqlab Biotechnologie GmbH, Erlangen, Germany).
Samples were then stored in double diluted $\mathrm{H}_{2} \mathrm{O}$ at $-20^{\circ} \mathrm{C}$.

\section{In silico analysis}

Based on in silico expression analyses with the EST profile viewer (National Center for Biotechnology Information (NCBI)), a strong overexpression of Sirt1 was observed in vascular endothelial cells. In addition, we had observed increased Sirt1 expression levels in response to the NO liberator sodium nitroprusside in earlier analyses [22]. Since VWF results from episodic reduction in peripheral blood flow in response to occupational exposure to hand-held vibrating machinery, and Sirt1 has been reported to promote endotheliumdependent vasodilation by targeting eNOS [21], we screened the Database of Single Nucleotide Polymorphisms (dbSNP; NCBI) [23], the HapMap database [24] and the Applied Biosystems database (TaqMan SNP Genotyping Assays, Applied Biosystems, Life Technologies Corporation, 5791 Van Allen Way, PO Box 6482 Carlsbad, California 92008 [25] for potential Sirt1 SNPs in order to further elucidate the role of Sirt1 in the pathogenesis of VWF. We focused our search criteria on coding nonsynonymous SNPs, in addition to a Sirt1 intron 4 SNP and a Sirt1 promoter SNP. As the HapMap data are publicly released to dbSNP, we performed our search mainly via dbSNP and the Applied Biosystems database, which both included HapMap data. 


\section{Genotyping}

Four putative genetic polymorphisms out of 113 within the Sirt1 genomic region (NCBI Gene Reference: NM_012238.3) were assessed on genomic DNA from patients with VWF and from healthy volunteers by real-time PCR. Allelic discrimination was performed by TaqMan-PCR-based allelespecific genotyping SNP-assays C_9638456_10 (NCBI SNP reference: rs1063114, exon 8), C_9638445_10 (NCBI SNP reference: rs1063111, exon 8), SIRT1-A485 (NCBI SNP reference: rs1063112, exon 8), C_25611590_10 (NCBI SNP reference: rs35224060, exon 9), C_27471644_10 (NCBI SNP reference: rs3740051, Sirt1 promoter) and C_15954063_10 (NCBI SNP reference: rs2236319, intron 4) as provided by the manufacturer (Applied Biosystems) (Table 2). PCR reactions and allele detection were done in duplicate and carried out in 96-well plates on an ABI PRISM 7000 Thermocycler (Applied Biosystems) and the ABI PRISM 7000 SDS software was used. For one PCR reaction (5 $\mu \mathrm{L} /$ well), $2.5 \mu \mathrm{L}$ TaqMan PCR Amplification Mix (2× TaqMan PCR Amplification Mix, No AmpErase UNG, Applied Biosystems) and $0.25 \mu \mathrm{L}$ of the 20× SNP Genotyping Assay Mix $(4 \mu \mathrm{M}$ probe, $18 \mu \mathrm{M}$ primer, Applied Biosystems) were added to each well and $2.25 \mu \mathrm{L}$ DNA (5 ng) were added. Every single run (96-well plate) included two no-template controls, a positive control (for Sirt1, BAC genomic clone RZPDB737C042021D) and 317 different DNA samples from healthy volunteers. After preincubation of the reaction mixture at $95^{\circ} \mathrm{C}$ for $10 \mathrm{~min}$, thermocycling was carried out at $92^{\circ} \mathrm{C}$ for $15 \mathrm{~s}$ and $60^{\circ} \mathrm{C}$ for $1 \mathrm{~min}$ for a total of 60 cycles. To discriminate allelic differences, fluorescence intensities of the reporter dyes VIC and FAM were read relative to the fluorescence intensity of a passive reference dye (ROX), which then yielded specific normalized reportervalues. In order to correct fluctuations in the optical quality of the reaction tubes that have been used, signal intensities that were measured prior to PCR were subtracted from the values that were measured after PCR (postread - preread).

\section{Competing interests}

The authors declare that they have no competing interests.

\section{Authors' contributions}

SVM conducted the study, designed and carried out the experiments, wrote the manuscript. BR carried out the experiments. CS carried out the experiments. CLD was responsible for the experimental design and statistical analysis. SL was responsible for the experimental design. UM was responsible for the study design, statistical analysis and writing of the manuscript. All authors read and approved the final manuscript.

\section{Acknowledgments}

This work was supported by institutional funds from the Saarland University Medical Center, Department of Internal Medicine, Division of Immunotherapy and Gene Therapy and the Johannes Gutenberg University Mainz, Medical School, Center for Preventive Medicine.

\section{Author details}

${ }^{1}$ Institute of Occupational, Social and Environmental Health, University of Mainz, Obere Zahlbacher Strasse 67, D-55131 Mainz, Germany. ${ }^{2}$ Institute of Occupational Medicine, Social Medicine and Health Services Research, University of Tuebingen, Wilhelmstrasse 27, D-72074 Tuebingen, Germany. ${ }^{3}$ Department of Internal Medicine, Division of Immunotherapy and Gene Therapy, José Carreras Research Center Saarland, University Medical Center, D-66421, Homburg/Saar, Germany.

Received: 25 April 2012 Accepted: 25 July 2012

Published: 1 October 2012

\section{References}

1. Palmer KT, Coggon D, Bendall HE, Pannett B, Griffin MJ, Haward BM: Handtransmitted vibration: occupational exposures and their health effects in Great Britain. Sudbury, Suffolk: HSE Books; 1999.

2. Bolognia JL, Jorizzo JL, Rapini RP: Dermatology: 2-Volume Set. St. Louis, MO: Mosby; 2007.

3. Taylor W: The vibration syndrome. London: Academic; 1974.

4. Dupuis H: Wirkung mechanischer Schwingungen auf das Hand-Arm-System. BAU Dortmund: Literaturanalyse; 1982.

5. Dupuis H, Riedel S: Handbuch der Arbeitsmedizin. In Vibrationsbedingtes Vasospastisches Syndrom VS (BK 2104). Edited by Konietzko J, Dupuis H. Landsberg: Ergomed Verlag; 1999:3-4. 2.

6. Dong C, Yoon W, Goldschmidt-Clermont P: DNA methylation and atherosclerosis. J Nutr 2002, 132:2406S-2409S.

7. Lund G, Andersson L, Lauria M, Lindholm M, Fraga MF, Villar-Garea A, Ballestar E, Esteller M, Zaina S: DNA methylation polymorphisms precede any histological sign of atherosclerosis in mice lacking apolipoprotein E. J Biol Chem 2004, 279:29147-29154.

8. Kim M, Kwon $H$, Lee $Y$, Baek JH, Jang JE, Lee SW, Moon EJ, Kim HS, Lee SK, Chung HY, Kim CW, Kim KW: Histone deacetylases induce angiogenesis by negative regulation of tumor suppressor genes. Nat Med 2001, 7:437-443.

9. Edelstein L, Pan A, Collins T: Chromatin modification and the endothelial-specific activation of the E-selectin gene. J Biol Chem 2005, 280:11192-11202.

10. Fish J, Matouk C, Rachlis A, Lin S, Tai SC, D'Abreo C, Marsden PA: The expression of endothelial nitric-oxide synthase is controlled by a cellspecific histone code. J Biol Chem 2002, 280:24824-24838.

11. Wu J, Iwata F, Grass J, Osborne CS, Elnitski L, Fraser P, Ohneda O, Yamamoto $\mathrm{M}$, Bresnick EH: Molecular determinants of NOTCH4 transcription in vascular endothelium. Mol Cell Biol 2005, 25:1458-1474.

12. Collins FS, Brooks LD, Chakravarti A: A DNA polymorphism discovery resource for research on human genetic variation. Genome Res 1998, 8:1229-1231.

13. Barnes MR: SNP and mutation data on the web - hidden treasures for uncovering. Comp Funct Genomics 2002, 3:67-74.

14. Zschoernig B, Mahlknecht U: SIRTUIN 1: regulating the regulator. Biochem Biophys Res Commun 2008, 376:251-255.

15. Zschoernig B, Mahlknecht U: Carboxy-terminal phosphorylation of SIRT1 by protein kinase CK2. Biochem Biophys Res Commun 2009, 381:372-377.

16. Mahlknecht U, Voelter-Mahlknecht S: Chromosomal characterization and localization of the NAD+-dependent histone deacetylase gene sirtuin 1 in the mouse. Int J Mol Med 2009, 23:245-252.

17. Voelter-Mahlknecht S, Mahlknecht U: Cloning, chromosomal characterization and mapping of the NAD-dependent histone deacetylases gene sirtuin 1. Int J Mol Med 2006, 17:59-67.

18. Blander G, Guarente L: The Sir2 family of protein deacetylases. Annu Rev Biochem 2004, 73:417-435.

19. Vaquero $A$, Scher $M$, Lee $D$, Erdjument-Bromage $H$, Tempst $P$, Reinberg $D$ : Human SirT1 interacts with histone $\mathrm{H} 1$ and promotes formation of facultative heterochromatin. Mol Cell 2004, 16:93-105.

20. Tai S, Robb G, Marsden P: Endothelial nitric oxide synthase: a new paradigm for gene regulation in the injured blood vessel. Arterioscler Thromb Vasc Biol 2004, 24:405-412.

21. Mattagajasingh I, Kim CS, Naqvi A, Yamamori T, Hoffman TA, Jung SB, DeRicco J, Kasuno K, Irani K: SIRT1 promotes endothelium-dependent vascular relaxation by activating endothelial nitric oxide synthase. Proc Natl Acad Sci U S A 2007, 104:14855-14860. 
22. Engel $\mathrm{N}$, Mahlknecht $\mathrm{U}$ : Aging and anti-aging: unexpected side effects of everyday medication through sirtuin 1 modulation. Int J Mol Med 2008, 21:223-232

23. Smigielski EM, Sirotkin K, Ward M, Sherry ST: dbSNP: a database of single nucleotide polymorphisms. Nucleic Acids Res 2000, 28:352-355.

24. International HapMap Consortium: The International HapMap Project. Nature 2003, 426:789-796.

25. De La Vega FM, Dailey D, Ziegle J, Williams J, Madden D, Gilbert DA: New generation pharmacogenomic tools: a SNP linkage disequilibrium Map, validated SNP assay resource, and high-throughput instrumentation system for large-scale genetic studies. Biotechniques 2002, 52:48-50. 54 .

doi:10.1186/1868-7083-4-18

Cite this article as: Voelter-Mahlknecht et al: Sirtuin 1 single nucleotide polymorphism (A2191G) is a diagnostic marker for vibration-induced white finger disease. Clinical Epigenetics 2012 4:18.

\section{Submit your next manuscript to BioMed Central and take full advantage of:}

- Convenient online submission

- Thorough peer review

- No space constraints or color figure charges

- Immediate publication on acceptance

- Inclusion in PubMed, CAS, Scopus and Google Scholar

- Research which is freely available for redistribution 